\title{
COVID-19 infection presenting as massive, multi-organ thromboembolism
}

\author{
Marco Bongiovanni ${ }^{1 *}$, Francesco Bini ${ }^{1}$, Bruno Dino Bodini ${ }^{1}$, Genti Xhepa ${ }^{2}$, Alessandro Maria Marra ${ }^{1}$ and Giuseppe De Angelis ${ }^{1}$ \\ ${ }^{1}$ Department of Medicine, ASST Rhodense, Garbagnate Milanese (Milan), Italy \\ ${ }^{2}$ Department of Radiology, ASST Rhodense, Garbagnate Milanese (Milan), Italy
}

\begin{abstract}
We report here the case of a 57-years old man presenting in the emergency department with chest and abdominal pain. Due to a familiar contact with a COVID-19 infected individual, a nasopharyngeal swab was done, resulting positive. ECG and blood tests were compatible with myocardial infarction. He underwent to coronaryangiography and angioplasty with implantation of medicated stent; dual antiplatelet therapy (acetylsalicylic acid and ticagrelor) and anticoagulant therapy (low molecular weight heparin) were started. A week later he developed acute pulmonary embolism and the next day sudden pain in the entire left lower limb which was cold and without peripheral arterial pulses; a thrombosis of the left common iliac artery, of the origin of the hypogastric artery and of the external iliac artery was diagnosed. Patient underwent to surgical embolectomy and was treated with the same antiplatelet and anticoagulant therapy following by complete symptoms remission.
\end{abstract}

\section{Introduction}

The emergence of Coronavirus disease 2019 (COVID-19) has presented an unprecedented challenge for the healthcare community across the world. Based on the rapid increase in the rate of human infection, the World Health Organization (WHO) has classified the COVID-19 outbreak as a pandemic [1-3]. Respiratory involvement, presenting as mild flu-like illness to potentially lethal acute respiratory distress syndrome or fulminant pneumonia, is the dominant clinical manifestation of COVID-19 [4]. However, pre-existing cardiovascular disease (CVD) and CV risk factors may enhance vulnerability to COVID-19; further, COVID-19 can worsen underlying CVD and even precipitate new cardiac complications, due to possible endothelial dysfunction [5-6]. Furthermore, patients with COVID-19 pneumonia exhibit a number of coagulation abnormalities that have been associated with a higher mortality rate [7-8]; in particular, severe lung inflammation and impaired pulmonary gas exchange in COVID-19 infected individuals has been suggested to be due to the up-regulation of pro-inflammatory cytokines [9]. Further, it has been demonstrated that the activation of the coagulation system is relevant in the pathogenesis of acute respiratory distress syndrome (ARDS), one of the most common complications of COVID-19 infection [10]. As a consequence, COVID-19 infection may predispose to both venous and arterial thromboembolic disease due to excessive inflammation, hypoxia, immobilization and diffuse intravascular coagulation (DIC).

We described here a case of a COVID-19 infected patient presenting with a massive, multi-organ thromboembolism (myocardial infarction, pulmonary embolism and systemic arterial embolism) treated with dual antiplatelet therapy and anticoagulation with low molecular weight heparin.

\section{Case report}

A 57 years old subject was evaluated at the Emergency Unit for chest, abdominal pain and vomiting arising the day before, associated with a week-long fever with anosmia and ageusia. He referred family contact for Covid-19 in the last few days. He was treated with sartanic and acetylsalicyclic acid for blood hypertension and was smoking addicted (20 pack/years). Blood tests showed significant increase of troponin (4786 ng/L), CPK (4357 IU/L), LDH (1152 IU/L), AST (695 $\mathrm{UI} / \mathrm{L})$ and d-dimer $(6720 \mathrm{ng} / \mathrm{ml})$ levels and low platelets count $(112,000$ cells/mmc). Mild hypocapnic hypoxemia with respiratory alkalosis was observed and $\mathrm{O}_{2}$ supply was administered. Nasopharyngeal swab for COVID-19 resulted positive, for which hydroxychloroquine $200 \mathrm{mg}$ x 2/day was set. ECG showed a ST lateral elevation in high lateral/lat seat with V1-V4 specularity; chest/abdomen CT with contrast showed bilateral interstitial thickening in the absence of other vascular, pleuroparenchymal and abdominal lesions (Figure 1a and 1c).

The patient underwent an emergency coronary-angiography which revealed floating thrombi in the proximal part of the anterior inter-ventricular artery and in the middle part of the right coronary in the absence of significant stenosis. Angioplasty performed with implantation of medicated stents. The patient was placed in dual antiplatelet therapy with acetylsalicylic acid and ticagrelor and anticoagulant therapy with low molecular weight heparin.

A week later the patient developed severe respiratory failure with hypoxemia and hypocapnia; on blood tests, evidence of significant increase in D-Dimer $(43.410 \mathrm{ng} / \mathrm{ml}), \mathrm{BNP}(3564 \mathrm{pg} / \mathrm{ml})$ and worsening of thrombocytopenia $(50,000$ cells/mmc). Systemic steroid therapy was set at a dose of $1 \mathrm{mg} / \mathrm{kg}$ of methylprednisolone; a chest CT scan with contrast was repeated showing a coarse thrombus in the auricle

*Correspondence to: AMarco Bongiovanni, Department of Medicine, ASST Rhodense, Garbagnate Milanese (Milan), Italy, Tel: +3902994303331; E-mail: mbongiovanni@asst-rhodense.it

Key words: COVID-19, thromboembolism, myocardial infarction

Received: December 02, 2020; Accepted: December 11, 2020; Published: December 14, 2020 


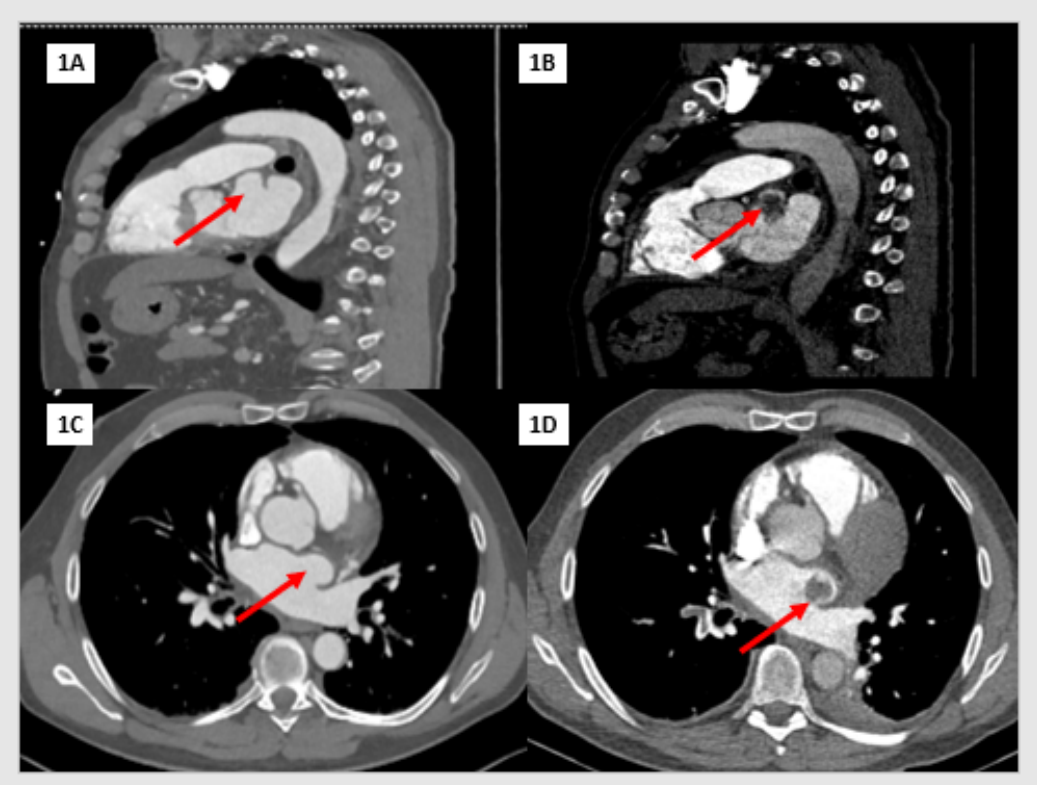

Figure 1. CT Scan of the Thorax in sagittal and coronal way at baseline (1A and 1C) and one week later (1B and 1D): arrows indicate the coarse thrombus that developed in the auricle of the left atrium

of the left atrium (Figure $1 \mathrm{~b}$ and $1 \mathrm{~d}$ ), associated with embolism in the right upper lobar artery and in its posterior segmental branch, in the right lower lobar artery and in its anterior and antero-external arterial branches. The next day the patient showed sudden pain in the entire left lower limb which appeared cold and with the complete absence of peripheral arterial pulses (femoral, popliteal and pedidium). Therefore, another CT scan with vessel's study of the abdomen and lower limbs was performed, showing vascular defects of thrombotic significance in the last part of the left common iliac artery with involvement of the origin of the hypogastric artery and in the external iliac artery which was occluded in its beginning and in the last part. Moreover, we observed a thrombosis at the middle-distal part of the popliteal artery, which had a tight caliber with involvement of the trifurcation and occlusion of the arteries of the leg. He underwent to surgical embolectomy according to Fogarty technique on left lower limb, with complete recovery of the functionality of the limb. Antithrombin III, lupus anticoagulant antibodies and omocystein levels were normal. Three days later, the patient recovered to normal respiratory parameters without oxygen supplementation. Blood tests showed normalization of CPK, AST and platelet values; $\mathrm{LDH}$, troponin and d-dimer levels significantly decreased. The same anti-aggregating and anticoagulant therapy were continued during all the hospitalization.

\section{Discussion and Conclusion}

Although respiratory findings are the dominant clinical manifestations of COVID-19, the shear burden of the illness implies that a large number of patients with COVID-19 would present with pre-existing CVD or develop new-onset cardiac dysfunction during the course of the illness. Further, both systemic venous and arterial thromboembolic disease are frequently observed in COVID-19 infected individuals. This clinical case confirms the high risk of CVD and thromboembolism in patients with COVID-19, especially in subjects with known underlying risk factors. The dramatic succession of events described, despite the administration of theoretically effective anti-platelet and anticoagulant treatment, highlights the procoagulant pattern of COVID-19 patients and the absolute need of a better description of the incidence, mechanisms, clinical management and outcomes of the thromboembolic diseases in these subjects.

\section{Declaration of competing interest}

The authors declare that they have no known competing financial interests or personal relationship that could have appeared to influence the work reported in this paper.

\section{References}

1. Chan JF-W, Yuan S, Kok K-H, To KK, Chu H, et al. (2020) A familial cluster of pneumonia associated with the 2019 novel coronavirus indicating person-to-person transmission: a study of a family cluster. Lancet 395: 514-523.

2. Rothe C, Schunk M, Sothmann P, Bretzel G, Froeschl G, et al. (2020) Transmission of SARS-CoV-2 infection from an asymptomatic contact in Germany. N Engl J Med 382: 970-971. [Crossref]

3. Holshue ML, DeBolt C, Lindquist S, Lofy KH, Wiesman J, et al. (2020) First case of 2019 novel coronavirus in the United States. N Engl J Med 382: 929-936. [Crossref]

4. Huang C, Wang Y, Li X, Ren L, Zhao J, et al. (2020) Clinical features of patients infected with 2019 novel coronavirus in Wuhan, China. Lancet 395: 497-506. [Crossref]

5. Shi S, Qin M, Shen B, Cai Y, Liu T, et al. (2020) Association of cardiac injury with mortality in hospitalized patients with COVID-19 in Wuhan, China. JAMA Cardiol 5: 802-810. [Crossref]

6. Xiong TY, Redwood S, Prendergast B, Chen M (2020) Coronaviruses and the cardiovascular system: acute and long-term implications. Eur Heart J 41: 1798-1800. [Crossref]

7. Klok FA, Kruip MJHA, van der Meer NJM, Arbous MS, Gommers DAMPJ, et al. (2020) Incidence of thrombotic complications in critically ill ICU patients with COVID-19. Thromb Res 191: 145-147. [Crossref]

8. Guan WJ, Ni ZY, Hu Y, Liang WH, Chen ZS, et al. (2020) Clinical characteristics of Coronavirus disease 2019 in China. $N$ Engl J Med 382: 1708-1720. [Crossref]

9. Zhou F, Yu T, Du R, Fan G, Liu Y, et al. (2020) Clinical course and risk factors for mortality of adult inpatients with COVID-19 in Wuhan, china: a retrospective cohort study. Lancet 395: 1054-1062.

10. Ozolina A, Sarkele M, Sabelnikovs O, Skesters A, Jaunalksne I, et al. (2016) Activation of coagulation and fibrinolysis in acute respiratory distress syndrome: a prospective pilot study. Front Med 3: 64.

Copyright: (C2020 Bongiovanni M. This is an open-access article distributed under the terms of the Creative Commons Attribution License, which permits unrestricted use, distribution, and reproduction in any medium, provided the original author and source are credited. 